\title{
Hybrid Transmission Schemes for Grouped Users in Cellular Systems
}

\author{
Juan F. Awad, Muhammad A. Imran and Rahim Tafazolli \\ Centre for Communication Systems Research \\ University of Surrey, Guildford, UK, GU2 7XH \\ Email: \{J.Awad, M.Imran, R.Tafazolli\}@ surrey.ac.uk
}

\begin{abstract}
Hybrid systems, where more than one transmission scheme are used within the same cluster, can be used as a way to improve spectral efficiency for the system as a whole and, more importantly, for the cell-edge users. In this paper, we will propose frequency reuse method by grouping the users into two groups, critical and non-critical users. Each user group is served with a transmission scheme, where the most vulnerable users are served by transmission scheme that avoid, make use of, and orthogonalise the interference. These schemes include the cooperative maximal ratio transmission and the non-cooperative orthogonal and non-orthogonal schemes. Radio resource allocation is studied and a solution is given for maximal ratio transmission and interference alignment. Simulation results are given, and showing the performance of each scheme when all users are considered critical and one scheme is used. Moreover,results showing the performance of our proposed frequency reuse scheme where different percentage of users considered critical.

Index Terms-Critical Users, Hybrid System, Maximal Ratio Transmission, Interference Alignment, Radio Resource Allocation.
\end{abstract}

\section{INTRODUCTION}

The ever increasing demand for higher data rates in mobile broadband communications and the scarcity of resources puts a lot of pressure on communication system designers. This is due to the rise of multimedia applications, VoIP and high speed Internet use in mobile devices. While users near the base station benefit from good channel conditions and high data rate, users at the cell edge or users who are in deep shadowing and fading will not be able to achieve such data rates.

OFDM has proven itself one of the most efficient multicarrier transmission techniques paving the way for the high data rates needed. This reputation comes from its ability to fight Inter-Symbol Interference (ISI) and combat frequency selectivity in wideband channels by using number of orthogonal low bandwidth subcarriers. Consequently, the multiple access version of OFDM was chosen for the downlink technique of the next generation mobile communications. While OFDM offers many benefit to the communication system, a proper distribution of subcarriers and available power between user is needed to achieve the best results. This is referred to as Radio Resource Allocation (RRA). RRA has been studied extensively in the literature, e.g. [1]-[3], due to its important role in improving the performance of wireless communication systems.
In this paper, we will propose a method to group users as cell-edge and cell-centre users. Each group is served using a different transmission scheme. This can be considered as a frequency reuse method different to other existing frequency reuse methods, like Fractional Frequency Reuse (FFR) [4] and Soft Frequency Reuse (SFR) [5]. Most frequency reuse schemes try to avoid the interference by orthogonalising or setting a power limit on interfering frequencies. Whereas here, we are trying to make good use of the interference generated by the surrounding base stations. cell-edge users, require transmission schemes that aim to avoid, orthogonalise, or make benefit from the interference. This is done in this paper by serving the cell-edge users using maximal ratio transmission or interference alignment where they use a sub-band orthogonal to that used by cell-centre users at the cell centre.

By considering multiple base station system as a Distributed-MIMO [6], we can benefit from the transmit diversity gain that can be achieve using Multiple-Input Single Output (MISO). Maximal Ratio Transmission (MRT) is a transmission scheme, [7], used in this paper to achieve this diversity gain [8]. In addition, we use interference alignment to orthogonalise the interference. Interference alignment is a linear precoding technique to align all the interference on one or more frequency, time or space dimensions. Although it has been recently found as a coding technique by [9], but then it was employed as a transmission scheme by the authors in [10] and [11]. Regardless of the number of the interferers, each user is able to access one half of the of the spectrum free from interference from other users [12].

The remainder of this paper will be organised as follows. In Section III we will be looking at the system model then formulate the resource allocation problem. In Section III the resource allocation problems of the maximal ratio transmission and the interference alignment is solved. Simulation results to show the system performance are provided in Section IV for both, simple and hybrid schemes. Finally, in Section $\nabla$ we conclude this paper.

\section{System Model AND Problem Formulation}

\section{A. System Model}

In a hexagonal cellular grid, we consider three sector downlink scenario each sector is serve with one Base Station (BS) antenna, as shown in Fig. 1. Each of the three BS connected together through a Central Processing Unit, on a backhaul of an 


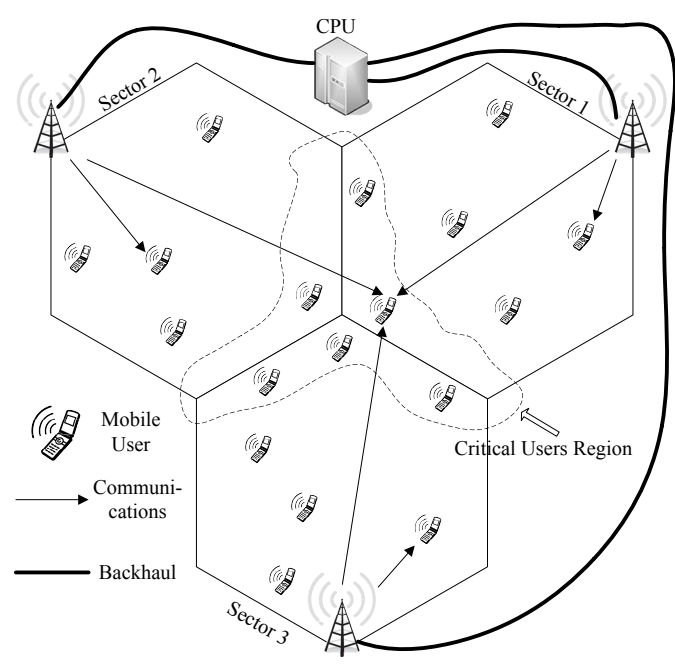

Fig. 1. System Model

unlimited bandwidth. The closest representation for this backhaul is an fibre optic backhaul. The job of the CPU is to control the cooperative part of the scheduling and resource allocation. This include choosing and assigning critical and non-critical users in each of the BS sectors alongside subcarrier allocation and power control for cooperative transmission schemes. Each BS forwards estimated channel state information to the CPU to be able to make informed resource allocation decisions. The Channel State Information (CSI) are considered to be estimated at the receiver and fed back to the BS fast enough to assume that the BS has full and instantaneous CSI. The channel is assumed to be frequency selective and the fading to be slow enough to remain constant within each transmission. OFDM is the chosen transmission technique to combat the frequency selectivity. In this paper, complexity is not the main concern, in fact, this paper focuses on improving spectral efficiency and fairness, and computational complexity will be given a secondary priority.

For most of this paper, all sectors are divided into two regions: the non-critical users region and the critical users region, as shown in Fig. 11 Each region is served using a different transmission scheme where non-critical users region is served using schemes with frequency reuse factor of 1 or 3 and the critical users region is served with interference combating schemes like orthogonal scheme with frequency reuse factor of 3 and cooperative schemes (i.e. IA and MRT). In our system model, users are chosen to be critical or noncritical on the basis of distance from their BS where critical users are the ones further away from the base station towards the centre of the cluster and and the rest are non-critical users.

We assume that each cluster is assigned a different frequency band and the interference from neighbouring clusters is small enough to be ignored. The available spectrum in each cluster is divided into two sub-bands, a sub-band for noncritical users region, $f^{n}$, and a sub-band for critical users region, $f^{c}$. Furthermore, each sub-band is used as a whole,
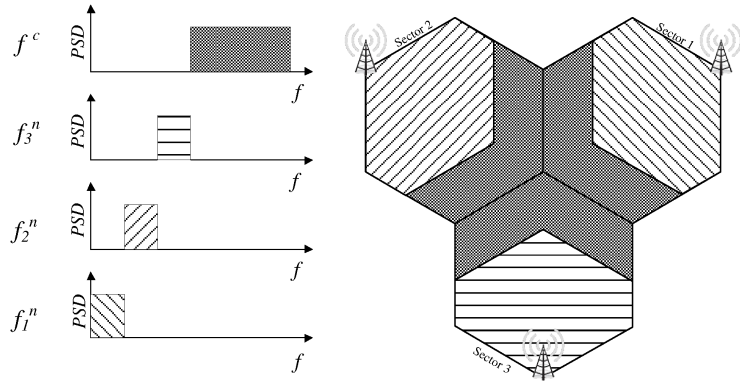

Fig. 2. Bandwidth Division

or divided, depending on the transmission scheme used in the corresponding region. In other words, if an orthogonal scheme with frequency reuse factor of 3 is used the sub-band is divided into three smaller and equal frequency bands, e.g. $f_{1}^{n}, f_{2}^{n}$ and $f_{3}^{n}$, whereas in a cooperative scheme (MRT and IA) or a nonorthogonal scheme with frequency reuse of factor 1, the same sub-band is used in all BSs. This frequency division scheme is illustrated in Fig. 2. In addition, the total power available at each BS is divided into power given to the users in the non-critical region, $P^{n}$, and users in the critical region, $P^{c}$, the total power of BS $m$ is $P_{m}^{T}=P_{m}^{n}+P_{m}^{c}$.

\section{B. Problem Formulation}

The main objective of this paper is to maximise the total sum rate of each sector in the case when a single transmission scheme is used or in the case of a hybrid system. This maximisation is constrained by the available resources (i.e. power and subcarriers) at each base station. The mathematical representation of this optimisation problem of the total sum rate of $\mathrm{BS} m$ is:

$$
\begin{aligned}
\max _{\mathbf{P}} & \sum_{k \in \mathcal{K}} R_{k} \\
\text { subject to : } & p_{k, n} \geq 0 \quad \forall n, k \in \mathcal{N}, \mathcal{K} \\
& \sum_{\mathcal{N}} \sum_{\mathcal{K}} p_{k, n} \leq P_{m}, \forall m,
\end{aligned}
$$

where $P_{m}$ is the total power available at BS $m . \mathcal{N}$ and $\mathcal{K}$ are the sets of users and subcarriers, respectively, available at their respective sector. $p_{k, n}$ is the transmit power to user $k$ on subcarrier $n$.

When hybrid transmission schemes are used the problem of the total sum rate becomes 


$$
\begin{aligned}
\max _{\mathbf{P}} & \sum_{k \in \mathcal{K}} R_{k}=\sum_{k \in \mathcal{K}^{n}} R_{k}^{n}+\sum_{k \in \mathcal{K}^{c}} R_{k}^{c} \\
\text { subject to : } \quad & p_{k, n} \geq 0 \quad \forall n, k \in \mathcal{N}^{n}, \mathcal{K}^{n} \\
& p_{k, n} \geq 0 \quad \forall n, k \in \mathcal{N}^{c}, \mathcal{K}^{c} \\
& \sum_{\mathcal{N}^{n}} \sum_{\mathcal{K}^{n}} p_{k, n} \leq P_{m}^{n}, \forall m \\
& \sum_{\mathcal{N}^{c}} \sum_{\mathcal{K}^{c}} p_{k, n} \leq P_{m}^{c}, \forall m,
\end{aligned}
$$

where $\sum_{k \in \mathcal{K}^{n}} R_{k}^{n}$ is the total sum rate of non-critical users using the rate calculation method $R_{k}^{n}$ depending on the transmission scheme used. Identically, the critical users' total sum rate is $\sum_{k \in \mathcal{K}^{c}} R_{k}^{c}$ using the specific transmission scheme rate calculation method $R_{k}^{c}$. $R_{k}^{c}$ and $R_{k}^{n}$ are different depending on the transmission scheme used for each user group. $\mathcal{K}^{n}$ and $\mathcal{K}^{c}$ are the sets of non-critical and critical users, respectively. Additionally, $\mathcal{N}^{n}$ and $\mathcal{N}^{c}$ are the sets of subcarriers assigned to non-critical and critical users regions, respectively. $P_{m}^{n}$ and $P_{m}^{c}$ are the total power available for the non-critical and the critical users regions in sector $m$, respectively.

Problem (2) can be decoupled into two independent problem, one for each transmission scheme, to reduce the complexity. This is possible by relaxing the coupling constraints of total power and total subcarriers. We achieve that by assigning them in a static manner according to the number of users in each region.

For the rest of this paper, a superscript of $n$ indicates a variable that corresponds to the non-critical users and a superscript of $c$ correspond to one related to the critical users.

\section{Resource Allocation for the Cooperative SCHEMES}

\section{A. Maximal Ratio Transmission}

The resource allocation problem for MRT is already shown in [13] and we will include it here in short for the sake of completeness.

1) Subcarriers Allocation: In maximal ratio transmission, each user should be connected to the three BSs at the same time using the same subcarrier so the signal can be added constructively at the receiver. In this case, subcarriers are assigned to users according to their average SNR from each of the three BSs. This is done in a greedy approach, where each user chooses the subcarrier that gives the highest average SNR. All users are given turn to choose and at the end no user is left without subcarrier. This method while being simple, it reduces the computational complexity on the CPU. It is worth noting that global channel knowledge is needed at each decision making terminal, in this case is the CPU.

2) Power Control: From [7] the rate of user $k$ is given by

$$
R_{k}=\sum_{n \in \mathcal{N}_{k}} B_{N} \log _{2}\left(1+\sum_{m \in \mathcal{M}} p_{k, n, m} \frac{\left|h_{k, n, m}\right|^{2}}{N_{0} B_{N}}\right),
$$

where $\mathcal{N}_{k}$ is the set of subcarriers assigned to user $k$ and $\mathcal{M}$ is the set of BSs. $p_{k, n, m}$ is the transmit power from the $m$ th antenna (i.e. BS) to the $k$ th user on the $n$th subcarrier, and $h_{k, n, m}$ is the channel from the $m$ th antenna (BS) to the $k$ th user on the $n$th subcarrier.

$$
\begin{aligned}
\max _{\mathbf{p}} & \sum_{k \in \mathcal{K}} R_{k} \\
\text { Subject to : } & p_{k, n, m} \geq 0 \quad \forall k, n, m \\
& \sum_{\mathcal{K}^{c}} \sum_{\mathcal{N}^{c}} p_{k, n, m} \leq P_{m}^{c} \quad \forall m,
\end{aligned}
$$

Applying Karush-Kuhn-Tucker (KKT) conditions, we get

$$
p_{k, n, m}=\left[\gamma-\frac{1}{H_{k, n, m}}\right]^{+} \quad \forall k, n, m,
$$

where $H_{k, n, m}=\frac{\left|h_{k, n, m}\right|^{2}}{N_{0} B / N}$ is the unit power SNR, $\gamma=$ $\left(\lambda_{1}+\ldots+\lambda_{M}\right)^{-1}$ is the water level and $[x]^{+}=\max (x, 0)$.

Eq. (5) look like a single-user water-filling at each BS, this will leads to significant reduction in computational complexity at the CPU.

\section{B. Interference Alignment}

Interference alignment applies to OFDM by considering the frequency bands (i.e. subcarriers) as symbol extensions in the frequency domain. Each subcarrier is considered a separate dimensions and for sufficiently large dimension we can achieve half of the interference-free degrees of freedom, assuming each user gives up half of its signal space. To achieve interference alignment, each of the three transmitters (BSs) and three of the users will constitute a three-user interference channel (IC). We solve the resource allocation problem of this sub-problem. Then, we choose another three users and define a different IC, so on until all feasible IC's are solved.

1) Subcarriers Allocation: A simplified algorithm for subcarrier allocation is proposed. A greedy approach is considered to assign subcarriers to users, where each user chooses the subcarrier with the best channel condition over all the other subcarriers. All users are given the chance to choose subcarriers, so no user is left without a subcarrier. The subcarrier allocation method is kept simple to keep in line with the subcarrier allocation methods used with other transmission schemes and mainly because the calculation of precoding and interference suppression matrices is a computationally extensive, and an optimal subcarrier allocation is prohibitive from the computational point of view. In this paper, we are aiming to achieve $3 / 2$ degrees of freedom for the three user interference channel requiring two transmit dimensions and two receive dimensions. That means, two subcarriers should be used for each transmission and the subcarrier allocation algorithm should assign even number of subcarriers for each user assuming the total number of subcarriers, $|\mathcal{N}|$, is even. Users are assigned pairs of subcarriers each from different one of the subsets $\mathcal{N}_{1}$ and $\mathcal{N}_{2}$. It is worth noting that $\left|\mathcal{N}_{1}\right|=\left|\mathcal{N}_{2}\right|$ and $\mathcal{N}_{1} \cap \mathcal{N}_{2}=\phi$ the channel from BS $m$ to user $k$ on subcarriers $n_{1}$ and $n_{2}$ is 


$$
H_{m k}^{n_{1} n_{2}}=\left[\begin{array}{cc}
h_{m k}^{n_{1}} & 0 \\
0 & h_{m k}^{n_{2}}
\end{array}\right],
$$

where $h_{m k}^{n_{1}}$ is the complex channel from BS $m$ to user $k$ on subcarriers $n_{1}$ and $h_{m k}^{n_{2}}$ on subcarrier $n_{2} . n_{1} \in \mathcal{N}_{1}$ and $n_{2} \in$ $\mathcal{N}_{2}$. The received signal at user $k$, in the three-user interference channel on the subcarriers $n_{1}$ and $n_{2}$ is

$$
Y_{k}=H_{k k}^{n_{1} n_{2}} X_{k}+H_{j_{1} k}^{n_{1} n_{2}} X_{j_{1}}+H_{j_{2} k}^{n_{1} n_{2}} X_{j_{2}}+Z_{k},
$$

where $X_{k}$ is the signal sent from transmitter (BS) $k$ and intended to user $k, Z_{k}$ is the received AWGN at user $k$. For the rest of this paper, we will drop the superscripts indicating the subcarrier indices.

2) Precoding and Interference Suppression: To eliminate the interference, the transmitted signal $X_{k}$ is pre-multiplied by a $2 \times 1$ precoding vector $V_{k}$ whose column(s) are the orthonormal basis of the signal space of the transmitter $k$. The received signal at receiver $k$ is given by:

$$
Y_{k}=H_{k k} V_{k} X_{k}+\sum_{j \neq k} H_{j k} V_{j} X_{j}+Z_{k} .
$$

Then we find the interference suppression vectors $U_{k}$ whose columns are the orthonormal basis of the interference free desired signal space. These are multiplied by the received signal at user $k$ :

$$
\bar{Y}_{k}=U_{k} H_{k k} V_{k} X_{k}+\sum_{j \neq k} U_{k} H_{j k} V_{j} X_{j}+U_{k} Z_{k},
$$

where $U_{k}$ is a $1 \times 2$ interference suppression vector and $\bar{Y}_{k}=$ $Y_{k} U_{k}$. The effective direct and interfering channels and noise resulting from equation (9) are:

$$
\begin{aligned}
h_{k k}^{1 \times 1} & =U_{k} H_{k k} V_{k}, \\
h_{j k}^{1 \times 1} & =U_{k} H_{j k} V_{j}, \\
z_{k} & =U_{k} Z_{k} .
\end{aligned}
$$

If the interference is aligned into the null space of $U_{k}$ then the following conditions hold:

$$
\begin{array}{r}
U_{k} H_{j k} V_{j}=\mathbf{0} \\
\operatorname{rank}\left(U_{k} H_{k k} V_{k}\right)=d_{k}
\end{array}
$$

$d_{k}$ is the requested degrees of freedom and the interference is completely eliminated.

The calculation of the vectors is not a straight forward process especially in finite dimensions, like in this scenario, where the number of subcarriers is finite. The authors in [10] gave an analytical method to calculate the precoding vectors leaving the problem of the interference suppression vectors open. Furthermore, the authors of [12] developed two iterative algorithms for the calculation of vectors. The first one is a leakage minimisation algorithm (leak-min) exploiting the channel reciprocity. The main property of this algorithm is that it is distributed with no need for centralised channel knowledge. The second algorithm works by maximising the received Signal-to-Interference-Ratio (max-SINR). Unlike the leak-min algorithm, this algorithm requires instantaneous and global channel. In [14], a rank constrained rank minimisation (RCRM) algorithm is developed considering an optimisation problem to minimise the dimension in which the interference spans with the constraints of the dimension of the signal space and it perform better than the previous algorithms in the case of frequency domain signal extension. A comparison between the three iterative algorithms in the case of symbol extension is shown in [14] where the RCRM algorithm performs best then the max-SINR followed by the leakage minimisation algorithm. In this paper, we will be using the leak-min algorithm despite its poor performance because it is distributed and, most importantly, is we can safely assume that the Residual Interference (RI) left at the receiver is negligible according to [15] to simply the power allocation problem.

3) Power Control: We define $\mathcal{N}^{p}=\left\{n_{1}^{p}, n_{2}^{p}, \ldots, n_{n_{c}}^{p}\right\}$ as the set of subcarrier pairs available for critical users.

From (9) and substituting in (10), (11) and (12), the rate of user $k$ operation under the Interference Alignment scheme defined as:

$$
R_{k}=\sum_{n^{p} \in \mathcal{N}_{k}^{p}} B_{N} \log _{2}\left(1+\frac{\left|h_{k k}^{n^{p}}\right|^{2}}{N_{0} B_{N}+\sum_{j \neq k}\left|h_{j k}^{n^{p}}\right|^{2}}\right) .
$$

Assuming the leakage minimisation algorithm has an optimal result, the interference received is eliminated and eq. (14) becomes:

$$
R_{k}=\sum_{n^{p} \in \mathcal{N}_{k}^{p}} B_{N} \log _{2}\left(1+\frac{\left|h_{k k}^{n^{p}}\right|^{2}}{N_{0} B_{N}}\right) .
$$

The maximisation problem for the power allocation in interference alignment scheme can now be formulated as follows:

$$
\begin{aligned}
& \max _{\mathbf{P}} \sum_{n^{p} \in \mathcal{N}_{k}^{p}} B_{N} \log _{2}\left(1+\frac{\left|h_{k k}^{n^{p}}\right|^{2}}{N_{0} B_{N}}\right) \\
& \text { subject to : } p_{k, n^{p}} \geq 0 \quad \forall n^{p}, k \in \mathcal{N}^{p}, \mathcal{K} \\
& \sum_{\mathcal{N}^{p}} \sum_{\mathcal{K}} p_{k, n^{p}} \leq P_{m}^{c},
\end{aligned}
$$

which is a convex problem and its optimal solution can be found using dual methods. The Lagrangian of eq. (16) is:

$\mathcal{L}(\mathbf{p}, \lambda, \nu)=-\sum_{\mathcal{K}} R_{k}+\lambda p_{k, n^{p}}-\nu\left(\sum_{\mathcal{N}^{p}} \sum_{\mathcal{K}} p_{k, n^{p}}-P_{m}^{c}\right)$.

By solving eq. (17) using the KKT conditions, the power assigned to subcarrier pair $n^{p}$ assigned to user $k$ becomes:

$$
p_{k, n^{p}}=\left[\frac{1}{\lambda}-\frac{1}{\tilde{H}_{k, n^{p}}}\right] \text {. }
$$


Here, $\lambda$ is the water level and $\tilde{H}_{k, n^{p}}=\frac{\left|h_{k k}^{n^{p}}\right|^{2}}{N_{0} B_{N}}$. Equation (18) is a water-filling equation at each BS. This means reduction on the computational load on the CPU and make our proposed system more distributed. The water-filling equation is found to be in line with parallel work given by the authors in [15].

\section{Simulation Results}

Our simulation results will be split into two main parts: Simple Schemes and Hybrid Schemes. The simulation model we are using here consists of three hexagonal sectors served by the three BSs located at the corners as shown in Figure 1 All simulation presented in this paper corresponds to a frequencyselective fading channel model according to ITU Pedestrian B [16]. The simulation parameters of both, simple and hybrid schemes, are given in Table II In both cases, the simulation is done over sufficiently large number of channel realisations.

TABLE I

SYSTEM PARAMETERS

\begin{tabular}{|c|c|c|}
\hline Parameter & Simple & Hybrid \\
\hline \hline Number of subcarriers & 128 & 128 \\
\hline Number of users in each sector & 12 & 12 \\
\hline Max power at Each Base Station & $50 \mathrm{~W}$ & $50 \mathrm{~W}$ \\
\hline Bandwidth & $20 \mathrm{MHz}$ & $20 \mathrm{MHz}$ \\
\hline Noise PSD & $-139 \mathrm{dBm}$ & $-139 \mathrm{dBm}$ \\
\hline Multipath Model & ITU Ped. B & ITU Ped. B \\
\hline Inter-Site Distance & 1 - 5km & $3 \mathrm{~km}$ \\
\hline Users' distribution & Co-Located & Hexagonal \\
\hline
\end{tabular}

\section{A. Simple Schemes}

In the simple scheme scenario all users are considered to be critical and it is done to give an insight into different transmission schemes and to identify which one could better serve the critical users. All users in this scenario are co-located together in the middle of the cluster at an equal distance from all the BSs. Although the users are all at the same place, they belongs to different BSs and assigned to different sectors.

We consider the capacity of single-user MIMO-OFDM system as an upper bound. The whole cluster is considered as single user MIMO with 3 transmit antennae and 36 receive antennae. No form of resource management is performed and the system capacity is, [17]:

$$
C=\frac{1}{M_{r} N} \mathbb{E}\left[\log _{2} \operatorname{det}\left(\mathbf{I}_{M_{r} N}+\frac{P_{\text {total }}}{M_{t} N\left(N_{0}\right)} \mathbf{H H}^{\dagger}\right)\right],
$$

given $\mathbf{H}$ as the block diagonal channel matrix. Each block represents the MIMO channel on one subcarrier between the base stations and the user terminals. $P_{\text {total }}$ is the total power of all BSs collectively, $M_{t}$ and $M_{r}$ is the number of transmit and receive antennae, respectively. $N$ is the total number of subcarriers and $\mathbf{I}_{M_{r} N}$ is the identity matrix of size $M_{r} N$. We can exploit the block-diagonal structure of the channel matrix

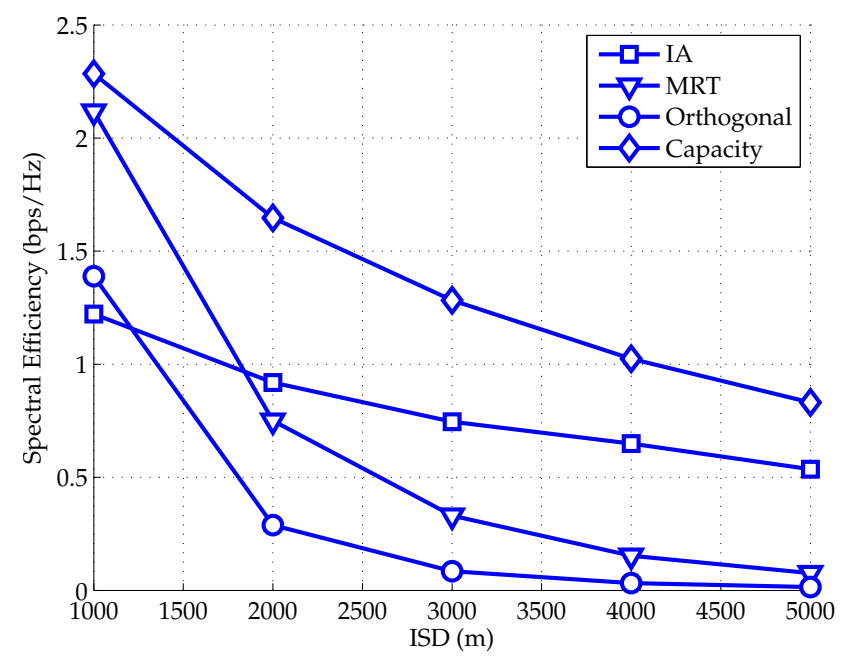

Fig. 3. Simple Schemes Comparison

to simplify (19) using the properties of determinants:

$$
C=\frac{1}{M_{r} N} \sum_{n=1}^{N} \mathbb{E}\left[\log _{2} \operatorname{det}\left(\mathbf{I}_{M_{r} N}+\frac{P_{\text {total }}}{M_{t} N\left(N_{0}\right)} \mathbf{H}_{n} \mathbf{H}_{n}^{\dagger}\right)\right],
$$

where $\mathbf{H}_{n}$ represent each subcarrier block from the original $\mathbf{H}$ given in eq. (19) Figure 3 shows a comparison between orthogonal frequency reuse factor of 3 , maximal ratio transmission and interference alignment and the MIMO-OFDM capacity, all as described in this paper where the spectral efficiency in $(\mathrm{bps} / \mathrm{Hz})$ is plotted against the inter-site distance (ISD) in meters. For small ISD we notice that the performance of MRT approaches the capacity and performs better than the orthogonal and the IA schemes whereas at high ISD the IA performs best over MRT and orthogonal and approaches capacity. This is due to the fact that in strong interference, the precoding and interference suppression vectors are unable to remove the interference, completely. Whereas in weak interference the performance of IA becomes better and approaches the $3 / 2$ degrees of freedom. It is also trivial for the MRT to outperforms the orthogonal scheme because of the added transmit diversity gain.

It worth reminding that MRT is not distributed whereas IA and the orthogonal scheme are distributed. The precoders for IA are calculated at the BS according to the leak-min algorithm used and no need for global channel knowledge. On the other hand, IA puts strains on the system in terms of computational complexity more than other transmission schemes.

\section{B. Hybrid Schemes}

In this scenario, we spread the users over the three sectors in a predetermined positions symmetrical between all sectors. The critical users' region is defined by the percentage of distance from the BS to the Clusters Mid-Point (CMD). For example, if we say that $20 \%$ of the sector is considered critical, it means that users located further than $80 \% \times($ Distance from BS to CMD) are considered to 


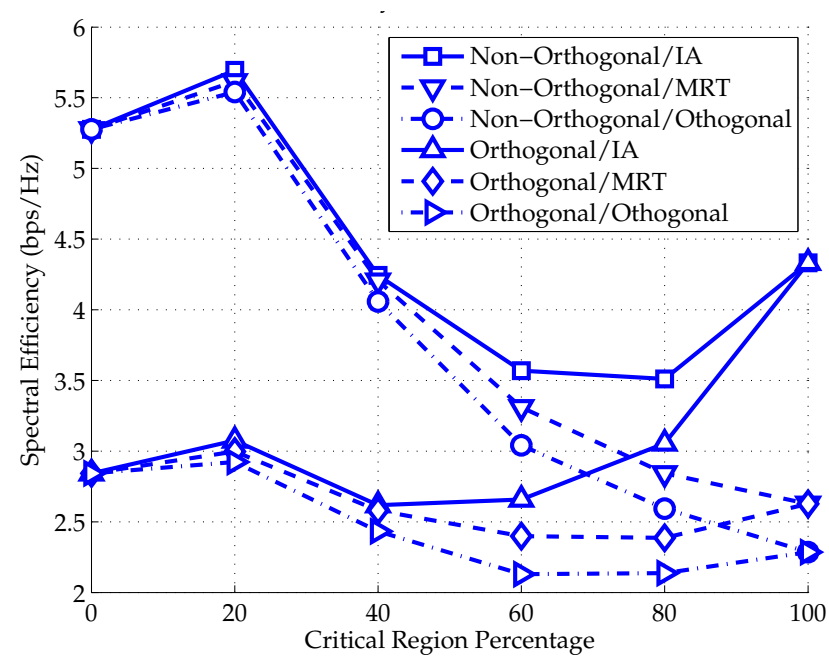

Fig. 4. Hybrid Schemes Comparison

be critical and the rest are non-critical. Furthermore, resources (subcarriers and power) is divided between the two regions with the same percentage.

Users are served using combinations of transmission schemes; orthogonal (frequency reuse factor of 3), maximal ratio transmission, interference alignment and, a nonorthogonal scheme (frequency reuse factor of) where interference caused by other users using the same sub-band is not ignored. Resource allocation for the non-orthogonal scheme is similar to that of orthogonal one, with greedy subcarrier allocation and single user water-filling power assignment. Non-critical users are served by the orthogonal or the nonorthogonal scheme because generally they have good channel condition and interference, for the non-orthogonal scheme, is weak. On the other hand, critical users require cooperative (and non-cooperative) schemes that avoid, make use of, and orthogonalise the interference generated elsewhere. So, MRT and IA serve the critical users and in addition to the orthogonal scheme.

In Figure 4 the performance of each hybrid scheme is plotted in spectral efficiency $(\mathrm{bps} / \mathrm{Hz}$ ) against the percentage of the critical users' region. We can easily notice the superiority of the non-orthogonal scheme over the orthogonal scheme for the non-critical users and that is due to the frequency reuse and weak interference. The point with the highest spectral efficiency is when $20 \%$ the sector is critical and is served by non-orthogonal and interference alignment schemes. The performance of the system degrades the bigger the critical region. Due to the greedy behaviour of the resource allocation methods, cooperative schemes turn into serving users with good channel conditions (near the BS), that means it will have bad channel conditions with other BSs causing the degradation. But when all of the user are critical, the performance rises due to the users who are in an extremely close proximity to the BSs having very good channel condition compensating the loss in the cooperation gain.

\section{CONClusion}

In this paper, we proposed a frequency reuse method, depends on the classifying users as critical and non-critical. Each class of users is served using a different transmission scheme. Vulnerable users served with transmission schemes that avoid, benefit from or orthogonalise the interference. Additionally, resource management solution is given for the cooperative transmission schemes, namely, maximal ratio transmission and interference alignment. Simulation results showed the performance of the system when all the users are critical. Alongside the system performance in hybrid scheme where results showed that best performance is achieved when $20 \%$ of users are considered to be critical.

\section{REFERENCES}

[1] J. Jang and K. Bok Lee, "Transmit power adaptation for multiuser OFDM systems," IEEE Journal on Slected Areas in Communications, vol. 21, no. 2, pp. 171-178, February 2003.

[2] W. Rhee and J. Cioffi, "Increase in capacity of multiuser OFDM system using dynamic subchannel allocation," in IEEE 51st Vehicular Technology Conference Proceedings, vol. 2, 2000, pp. 1085-1089.

[3] S. Sadr, A. Anpalagan, and K. Raahemifar, "Radio resource allocation algorithms for the downlink of multiuser ofdm communication systems," IEEE Communications Surveys Tutorials, vol. 11, no. 3, pp. 92-106, Third Quarter 2009.

[4] M. Sternad, T. Ottosson, A. Ahlen, and A. Svensson, "Attaining both coverage and spectral efficiency with adaptive OFDM downlinks," in Proceedings of The 58th IEEE Semiannua Vehicular Technology Conference, 2003. VTC 2003-Fall., October 2003.

[5] Huawei, Soft Frequency Reuse Scheme. UTRAN LTE, 3GPP R1050507, TSG RAN WG1 Meeting 41, Athens, Greece, May 2007.

[6] J. Wang, F. Adachi, and X. Xia, "Coordinated and distributed mimo [guest editorial]," IEEE Wireless Communications, vol. 17, no. 3, pp. 24-25, June 2010.

[7] A. Goldsmith, Wireless Communications. Cambridge University Press, 2005.

[8] J. Cavers, "Single-user and multiuser adaptive maximal ratio transmission for Rayleigh channels," IEEE Transactions on Vehicular Technology, vol. 49, no. 6, pp. 2043-2050, Nov 2000.

[9] M. Maddah-Ali, A. Motahari, and A. Khandani, "Signaling over mimo multi-base systems: Combination of multi-access and broadcast schemes," in Information Theory, 2006 IEEE International Symposium on, Jul. 2006, pp. 2104-2108.

[10] V. Cadambe and S. Jafar, "Interference alignment and degrees of freedom of the $K$-user interference channel," Information Theory, IEEE Transactions on, vol. 54, no. 8, pp. 3425-3441, Aug. 2008.

[11] _ - "Interference alignment and the degrees of freedom of wireless $X$-networks," Information Theory, IEEE Transactions on, vol. 55, no. 9, pp. 3893-3908, Sept. 2009.

[12] K. Gomadam, V. Cadambe, and S. Jafar, "A distributed numerical approach to interference alignment and applications to wireless interference networks," Information Theory, IEEE Transactions on, vol. 57, no. 6, pp. 3309-3322, Jun. 2011.

[13] J. Awad, M. Imran, and R. Tafazolli, "Improving fairness by cooperative communications and selection of critical users," in Wireless Communications and Mobile Computing Conference (IWCMC), 2011 7th International, July 2011, pp. 184-188.

[14] D. S. Papailiopoulos and A. G. Dimakis, "Interference alignment as a rank constrained rank minimization," arXiv.org, vol. abs/1010.0476, 2010, http://arxiv.org/abs/1010.0476

[15] B. Da and R. Zhang, "Exploiting interference alignment in multi-cell cooperative ofdma resource allocation," in IEEE Global Telecommunications Conference (GLOBECOM 2011), Dec. 2011, pp. 1-5.

[16] "Recommendation ITU-R M.1225: Guidelines for evaluation of radio transmission technologies for IMT-2000," Tech. Rep., 1997.

[17] S. Chatzinotas, M. Imran, and R. Hoshyar, "On the ergodic capacity of the wideband mimo channel," in Vehicular Technology Conference, 2009. VTC Spring 2009. IEEE 69th, april 2009, pp. 1-5. 